\title{
Modal Analysis of Semiconductor Lasers with Nonplanar Mirrors
}

\author{
ROBERT J. LANG, STUDENT MEMBER, IEEE, JOSEPH SALZMAN, AND AMNON YARIV, FELLOW, IEEE
}

\begin{abstract}
We present a formalism for analyzing laser resonators which possèss nonplanar mirrors and lateral waveguiding [e.g., an unstable resonator semiconductor laser (URSL)]. The electric field is expanded in lateral modes of the complex-index waveguide and is required to reproduce itself after one roundtrip of the cavity. We show how the waveguide modes, their gain and loss, and hence the criterion for truncation of the infinite set of modes can be derived from the Green's function of the one-dimensional eigenvalue equation for the waveguide. Examples are presented for three cases of interest-a purely gain-guided URSL, an index-guided URSL, and a gain-guided tiltedmirror resonator. We compare theoretical calculations to previous experiments.
\end{abstract}

\section{I. ÍNTRODUCTION}

$\mathrm{O}$ VER the past 20 years, semiconductor lasers have evolved into a reliable source of coherent, near-IR light. Their small size and low power requirement have made them the subject of intense study for use in communications systems. However, the small size of semiconductor lasers limits the available output power since the high intensities at the output faces leads to catastrophic facet damage and laser failure at higher current levels.

This problem can be alleviated by increasing the size of the emitting area. One means of accomplishing this is to increase the transverse dimension of the laser (e.g., a large optical cavity (LOC) structure [1]). However, the transverse dimension can only be increased up to about 1 $\mu \mathrm{m}$, at which point, progressively, additional transverse modes appear resulting in a loss of coherence.

Another means of increasing the emitting area is to enlarge the lateral dimension of the laser. However, as the width exceeds about $10 \mu \mathrm{m}$, the phenomenon of filamentation or regenerative self-focusing appears. The positions of the individual filaments are unstable and shift about under current modulation. This produces unpredictable kinks in the light-current curve and reduces the coherence of the laser light.

To date, there have been two different approaches to the problem of increasing the width (and consequently the power) of semiconductor lasers. One has been to fabricate

Manuscript received July 30, 1985; revised October 7, 1985. This work was supported by grants from Rockwell International, the Air Force Office of Scientific Research, and the Office of Naval Research. The work of R. Lang and J. Salzman was supported by the National Science Foundation and a Bantrell Post-Doctoral Fellowship.

The authors are with the Department of Applied Physics, California Institute of Technology, Pasadena, CA 91125.

IEEE Log Number 8407059. phase-locked arrays of lasers which are individually too narrow for filamentation to appear [2]. Such lasers have produced the highest $\mathrm{CW}$ power reported to date [3]; however, their design remains plagued by problems of incomplete phase-locking [4] and the appearance of an antisymmetric far field [5].

The second approach has been to fabricate broad-area lasers with nonplanar mirrors. Although the advantages of high-loss resonators for lasing media with high gain have been recognized for years, it is only recently that there have been attempts to apply such resonators to semiconductor lasers [6]-[8]. Unstable resonators, resonators which possess a magnification greater than 1.0 , possess large mode volumes [9], enabling them to effectively utilize all of the lasing volume. They can have high discrimination against higher order modes and are thus less sensitive to material imperfections, and because of their magnifying abilities they tend to suppress filamentation [8].

The properties of unstable resonators, in particular, have been described extensively in the literature. However, the application of such theory to semiconductor lasers encounters several problems which stem from the unique character of semiconductor lasers. Most theories [9]-[12], [20] treat the resonator as consisting of two mirrors bounding a homogeneous medium; yet gain guiding plays a substantial role in semiconductor lasers. Secondly, the finite size of the mirrors introduces effects due to edge diffraction that are lost in theories which assume infinite mirrors [9], [21]. The two-dimensional nature of the semiconductor laser suggests many asymmetric configurations which would be impossible in a three-dimensional laser, and consequently, have not been analyzed. Also, the lossy material surrounding the pumped region absorbs the diffracted wave, which suggests that a real index waveguide in the lateral direction could increase the quantum efficiency of a URSL [12]. Finally, there are many geometries other than unstable resonators (e.g., a tilted-mirror resonator [13] or a hybrid URSL/FabryPerot resonator [6]) which show promise of high power, yet have not been analyzed.

The foregoing considerations show the need for a method of analyzing broad-area semiconductor lasers with nonplanar mirrors. We present in this paper a general solution to this problem. In Section II, we outline the mirror-coupled mode formalism. In Section III, we derive the complex waveguide modes to be used as a basis set and 
justify the truncation of the set to a finite size. In Section IV, we calculate the coupling coefficients which characterize the mirrors and show how the nonlinear eigenvalue equation can be simplified to a linear eigenvalue problem. In Section V, we present results for three specific cases: a gain-guided URSL, an index-guided URSL, and a tilted-mirror resonator. We conclude in Section VI by summarizing the important results of the analysis.

\section{Outline of the Formalism}

The device we are considering is shown schematically in Fig. 1. It consists of a symmetric lateral waveguide with (complex) indexes of refraction $n_{1}$ (cladding) and $n_{0}$ (core), terminated by nonplanar mirrors $R_{1}$ and $R_{2}$. We assume that all transverse ( $x$ axis) variation has been removed using an effective index approximation [14]. The electric field can be written in terms of the complete set of modes of the lateral waveguide as

$$
E_{y}(y, z)=\sum_{n} a_{n} E_{n}(y) e^{-j \beta_{n} z}
$$

where the $\left\{a_{n}\right\}$ and $\left\{\beta_{n}\right\}$ are the complex mode amplitudes and propagation constants, respectively, of the (also complex) modes $\left\{E_{n}\right\}$ of the lateral waveguide. The sum in (1) is assumed to include the integration over continuum modes (radiation modes), which must be included for completeness. We can represent the field $E_{y}(y, z)$ by a column vector $\tilde{A}$ with components $\left\{a_{n}\right\}$; then the result of a propagation through a distance $L$ within the waveguide can be expressed by left multiplying by a propagation matrix

$$
\tilde{\tilde{P}}(L)=e^{-j \bar{B} L}
$$

where the $n, m$ component of $\tilde{\tilde{B}}$ is $\delta_{n m} \beta_{n}$.

We model each mirror by a complex reflectivity $R(y)$, so that the field immediately after reflection is $R(y) E_{y}(y$, $z=L)$. Since the set of $\left\{E_{n}(y)\right\}$ is complete, we can expand the field after reflection in terms of the $\left\{E_{n}\right\}$; in particular, a mode $E_{n}(y)$ after reflection can be written

$$
R(y) E_{n}(y)=\sum_{m} r_{n m} E_{m}(y)
$$

where $\left\{r_{n m}\right\}$ are the elements of a mirror coupling matrix $\tilde{\tilde{R}}$ given by

$$
r_{n m} \equiv\left(E_{m}^{\dagger}(y), \quad R(y) E_{n}(y)\right) .
$$

$\left\{E_{m}^{\dagger}\right\}$ are the eigenmodes of the adjoint operator and (,) signifies the appropriate inner product.

The eigenmodes of the resonator are found by requiring that the lasing field reproduce itself after one roundtrip, i.e., that

$$
\tilde{\tilde{R}}_{2} \tilde{\tilde{P}}(L) \tilde{\tilde{R}} \tilde{\tilde{P}}(L) \tilde{A}=\tilde{A} .
$$

This is, in general, a nonlinear eigenvalue problem. The free parameters $[\omega$, the lasing frequency, and $n$, the threshold carrier density (or equivalently threshold gain)] are imbedded within the matrix $\tilde{\tilde{P}}(L)$ via the dependence of the propagation constants $\beta_{n}$ upon $\omega$ and $n$, and to a

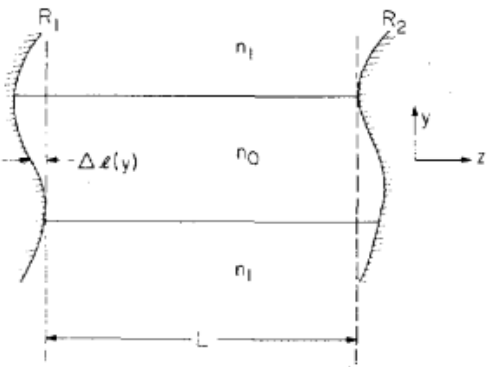

Fig. 1. Schematic of a broad-area laser with nonplanar mirrors and a lateral waveguide.

lesser extent, within the reflectivity matrices due to the dependence of the modes themselves upon $\omega$ and $n$. As it stands, the solution to (5) is not immediately forthcoming. Besides the nonlinearity, the matrices are infinite, and in places, continuous. In the next two sections, we will introduce and justify the approximations necessary to reduce the problem of solving (5) to a manageable task.

\section{Lateral Waveguide Modes}

Our first task is to truncate the set of lateral waveguide modes to some manageable number (according to some rational criterion) and to deal with the troublesome continuum modes. The modes of a symmetric real index waveguide are well known [15]; however, some complications occur when we open up the field to include a complex index of refraction (i.e., gain/loss). There is also the question of whether to include "leaky" modes (which diverge as $y \rightarrow \pm \infty$ ) in our basis. Furthermore, since we are dealing with a complex potential, the problem is nonself-adjoint, and the orthogonality condition between modes is different from that of real index-guided modes. We shall deal with all of these issues in one fell swoop by deriving the complex waveguide modes from the original equation. In the process, a simple picture of the analytic structure of the Green's function for the lateral mode eigenvalue equation will show which modes to keep and which we can safely discard.

We begin with the Helmholtz equation for TE waves in the structure shown in Fig. 1.

$$
\left[\nabla_{y z}^{2}+\frac{\omega^{2} n^{2}(y)}{c^{2}}\right] E_{y}(y, z)=0
$$

where $\omega$ is the oscillation frequency, $c$ is the speed of light, and

$$
n(y)= \begin{cases}n_{0} & |y| \leq d \\ n_{1} & |y|>d .\end{cases}
$$

We choose our time factor as $e^{j \omega t}$ and search for solutions of the form

$$
E_{y}(y, z)=e^{-j \beta z} u(\zeta), \quad \zeta \equiv y / d,
$$

and since we are looking for positive $z$-traveling waves, we stipulate

$$
\operatorname{Re}(\beta) \geq 0 \text {. }
$$


We define

$$
\begin{aligned}
v(\zeta) & \equiv\left[\frac{\omega d n(\zeta d)}{c}\right]^{2}, \quad v_{0} \equiv\left[\frac{\omega d n_{0}}{c}\right]^{2}, \\
v_{1} & \equiv\left[\frac{\omega d n_{1}}{c}\right]^{2}, \quad \lambda \equiv-[\beta d]^{2}
\end{aligned}
$$

to arrive at the nondimensional equation

$$
\left[\frac{d^{2}}{d \zeta^{2}}+v(\zeta)\right] u(\zeta)+\lambda u(\zeta)=0
$$

The problem is fully specified when we include boundary conditions. It is convenient to work out even and odd fields separately (it is clear that any field can be decomposed into an even part and an odd part); so we can solve (11) on the interval $\zeta \in(0, \infty)$ with the boundary conditions

$$
u^{\prime}(0)=0, \quad u \text { bounded at } \zeta=\infty
$$

for even modes and

$$
u(0)=0, \quad u \text { bounded at } \zeta=\infty
$$

for odd modes.

A well-known result of spectral theory [16] is that the appropriate spectral representation for an operator $L$ of the equation

$$
L u+\lambda u=0
$$

can be determined by integrating the Green's function for the operator on a great circle in the complex $\lambda$ plane. In this process, nonanalyticities give rise to the spectrum; poles generate a discrete spectrum, and branch cuts give a continuous spectrum. The Green's function, of course, is the solution to

$$
[L+\lambda] G(\zeta, \eta ; \lambda)=\delta(\zeta-\eta) .
$$

For the even modes, the Green's function is easily verified by direct substitution to be

$G(\zeta, \eta ; \lambda)$

$$
=\frac{u_{L}\left(\zeta_{<}\right) u_{R}\left(\zeta_{>}\right)}{\sqrt{\lambda+v_{0}} \sin \sqrt{\lambda+v_{0}}-j \sqrt{\lambda+v_{1}} \cos \sqrt{\lambda+v_{0}}}
$$

with

$$
\begin{aligned}
\zeta<\equiv \min (\zeta, \eta) \\
\zeta_{>} \equiv \max (\zeta, \eta) \\
u_{L}(\zeta) \equiv \begin{cases}\cos \zeta \sqrt{\lambda+v_{0}}, \quad \zeta \leq 1 \\
\cos \sqrt{\lambda+v_{0}} \cos (\zeta-1) \sqrt{\lambda+v_{1}} & \\
-\sqrt{\frac{\lambda+v_{1}}{\lambda+v_{0}}} \sin (\zeta-1) \sqrt{\lambda+v_{1}}, & \zeta>1\end{cases}
\end{aligned}
$$

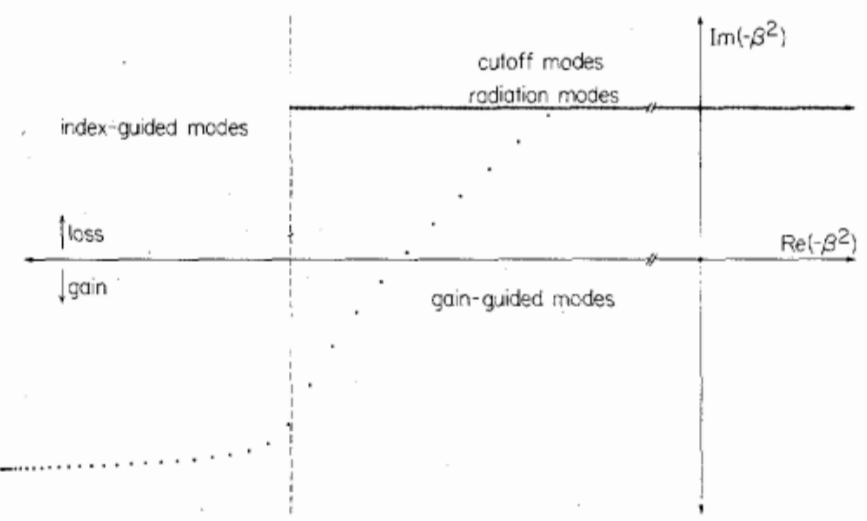

Fig. 2. Analytic structure in the complex $\lambda$ plane of the Green's function for the even waveguide modes of a waveguide of width $20 \mu \mathrm{m}$; core: $n$ $=3.50$, gain $=50 \mathrm{~cm}^{-1}$, cladding: $n=3.40$, loss $=50 \mathrm{~cm}^{-1}$. *'s are poles (corresponding to discrete modes), while the cross-hatched line indicates a branch cut (continuum modes).

$u_{R}(\zeta) \equiv\left\{\begin{array}{lc}\cos (\zeta-1) \sqrt{\lambda+v_{0}}-j \sqrt{\frac{\lambda+v_{1}}{\lambda+v_{0}}} \\ \sin (\zeta-1) \sqrt{\lambda+v_{1}}, & \zeta \leq 1 \\ \exp -j(\zeta-1) \sqrt{\lambda+v_{1}}, & \zeta>1\end{array}\right.$

Anticipating the great circle integral, we examine the analytic structure of $G$ in the complex $\lambda$ plane. Clearly, there are poles wherever

$$
\sqrt{\lambda+v_{0}} \sin \sqrt{\lambda+v_{0}}-j \sqrt{\lambda+v_{1}} \cos \sqrt{\lambda+v_{0}}=0
$$

which will give rise to discrete modes. In addition, there is a branch cut emanating from $\lambda=-v_{1}$. (The quantity $\sqrt{\lambda+v_{0}}$ appears only in even powers; hence, there is no branch cut.) The direction of the branch cut can be inferred from the boundedness of $u$; this, plus the last line of (16), imply

$$
\operatorname{Im} \sqrt{\lambda+v_{1}} \leq 0, \quad \text { so }-2 \pi \leq \arg \left(\lambda+v_{1}\right) \leq 0 .
$$

This structure is illustrated in Fig. 2. Each nonanalyticity corresponds to a mode of the waveguide with a propagation constant $\beta$ given by $\beta=d^{-1} \sqrt{-\lambda}, \operatorname{Re} \beta \geq$ 0 . Near the negative real axis, a positive imaginary part of $\lambda$ yields a negative imaginary part for $\beta$, implying a lossy mode. From this picture, we can compare the losses of all of the waveguide modes using the qualitative rule up $=$ loss, down $=$ gain. To aid in the interpretation of the different modes, modes in different regions of the plane have been labeled in accordance with common usage.

Radiation modes (corresponding to the branch cut) present a special problem to a numerical solution of (5) because they are continuous. They can be dealt with by the following simple expedient:

$$
\text { We shall ignore all continuum modes. }
$$


The question is, are we justified in doing so? The resonator modes we are interested in are those with the lowest loss; consequently, our intuition suggests that we should use low-loss waveguide modes as a basis set. A closer examination shows that over the length of the laser, the continuum modes are strongly attenuated compared to the lower loss discrete modes; consequently, they make negligible contribution to the resonator mode after propagation and can, in fact, be ignored. Furthermore, any discrete modes lying above the branch cut have even higher loss than the continuum modes, so we gain nothing by including them in the basis set. Finally, although the 'gain-guided"' modes are beyond what is traditionally thought of as cutoff $\left(-\lambda<\operatorname{Re} v_{0}\right)$, their loss is comparable to the index-guided modes (if any exist) and should be included.

The validity of the above argument relies on the vertical separation between $v_{0}$ and $v_{1}$ in the $\lambda$-plane, that is, upon the high gain of the core and/or the high loss of the cladding. This pictorial representation indicates the validity of the approximation-for a low-gain medium (e.g., a gas laser) or purely real index guiding, both $v_{0}$ and $v_{1}$ lie on the real axis. There is no gain separation between continuum and discrete modes and the theory breaks down. For semiconductor lasers, however, with gains on the order of $50 \mathrm{~cm}^{-1}$ or greater, the gain separation is large enough that neglecting continuum modes is a good approximation. (It should be noted that for high-loss resonators, the gain is necessarily much larger than for Fabry-Perot resonators, which strengthens the approximation.)

To summarize, we will use only those discrete modes which lie below the branch cut in Fig. 2. Carrying out the great circle integration yields

$$
u_{n}(\zeta) \equiv\left\{\begin{array}{c}
\cos \zeta \sqrt{\lambda_{n}+v_{0}}, \quad 0 \leq|\zeta| \leq 1 \\
\cos \sqrt{\lambda_{n}+v_{0}} \exp -j(|\zeta|-1) \sqrt{\lambda_{n}+v_{1}}, \\
|\zeta| \geq 1
\end{array}\right.
$$

with the orthogonality condition

$$
\int_{0}^{\infty} d \zeta u_{n}(\zeta) u_{m}(\zeta)=\frac{1}{2} \delta_{n m}\left(1+\frac{1}{j \sqrt{\lambda_{n}+v_{1}}}\right)
$$

and the $\left\{\lambda_{n}\right\}$ satisfy

$$
\begin{aligned}
& \sqrt{\lambda_{n}+v_{0}} \sin \sqrt{\lambda_{n}+v_{0}} \\
& -j \sqrt{\lambda_{n}+v_{1}} \cos \sqrt{\lambda_{n}+v_{0}}=0,
\end{aligned}
$$

$\operatorname{Im} \lambda_{n} \leq \operatorname{Im}-v_{1}, \quad-2 \pi \leq \arg \left(\lambda_{n}+v_{1}\right) \leq 0$.

A similar procedure for the odd modes yields

$$
u_{n}(\zeta) \equiv\left\{\begin{array}{c}
\sin \zeta \sqrt{\lambda_{n}+v_{0}}, \quad 0 \leq|\zeta| \leq 1 \\
\sin \sqrt{\lambda_{n}+v_{0}} \exp -j(|\zeta|-1) \sqrt{\lambda_{n}+v_{0}}, \\
|\zeta| \geq 1
\end{array}\right.
$$

where the $\left\{\lambda_{n}\right\}$ satisfv

$$
\begin{aligned}
& \sqrt{\lambda_{n}}+v_{0} \cos \sqrt{\lambda_{n}+v_{0}} \\
& +j \sqrt{\lambda_{n}+v_{1}} \sin \sqrt{\lambda_{n}+v_{0}}=0
\end{aligned}
$$

and the $\left\{u_{n}\right\}$ satisfy the same orthogonality condition. (Obviously, any even mode is orthogonal to any odd mode.) A comparison of (20) and (23) to the standard theory of real dielectric waveguides reveals that they are precisely the dispersion relations for real index waveguides, extended into the complex plane.

\section{Mirror Coupling and Diagonalization}

Referring back to Fig. 1, we see that the mirror reflectivity $R(y)$ is the reflectivity seen at a fixed reference plane. The common thin-lens approximation [17] is to model a nonplanar reflector as a complex phase shift of $-2 j \beta \Delta l(y)$ where $\beta$ is the material propagation constant and $\Delta l(y)$ is the distance from the mirror to the reference plane. The dielectric reflectivity and/or scattering can be included by an additional multiplying factor $r(y)$. A single waveguide mode $u_{n}(\zeta)$ upon reflection can be reexpressed as a sum of waveguide modes:

$$
r(y) e^{-2 j \beta(y) \Delta l(y)} u_{n}(\zeta)=\sum_{m} r_{n m} u_{m}(\zeta) .
$$

Multiplying by $u_{m}(\zeta)$ and integrating yields

$$
\begin{aligned}
r_{n m}= & \left(1+\frac{1}{j \sqrt{\lambda_{m}+v_{1}}}\right)^{-1} \int_{\text {mirror }} d \zeta\left\{r(\zeta d) u_{m}(\zeta)\right. \\
& \left.\cdot \exp [-2 j \sqrt{v(\zeta)} \Delta l(\zeta d) / d] u_{n}(\zeta)\right\}
\end{aligned}
$$

where the relation $y=\zeta d$ has been used. Within the various regions of the waveguide, $u_{n}$ and $u_{m}$ consist of linear exponentials; most mirrors of interest are piecewise linear or (to a good approximation) quadratic; consequently, all of the mirror coupling coefficients are, at worst, sums of complex error functions for which simple approximations are readily available [18]. For example, for a curved mirror of radius $-R$ with unity reflectivity which extends only to the edge of the waveguide, we find

$$
\begin{aligned}
r_{n m}= & \left(1+\frac{1}{j \sqrt{\lambda_{m}+v_{1}}}\right)^{-1} \\
& \cdot \int_{-1}^{+1} d \zeta \cos \left(\frac{t_{m} \pi}{2}-\zeta \sqrt{\lambda_{m}+v_{0}}\right) \\
& \cdot \cos \left(\frac{t_{n} \pi}{2}-\zeta \sqrt{\lambda_{n}+v_{0}}\right) \exp \left[-j \sqrt{v_{0}} \zeta^{2} d / R\right]
\end{aligned}
$$

where $t_{i}=1$ if the $i$ th mode is odd and 0 if it is even. (A more accurate model would include the spatial and angular variation of the reflectivity within the integral. We have assumed constant reflectivity throughout this work.)

We are left with the task of simplifying the frequency and gain dependence of the matrices. We perform a per- 
turbation expansion around a fixed operating point

$$
n \rightarrow \bar{n}+\Delta n, \quad \omega \rightarrow \bar{\omega}+\Delta \omega
$$

and define

$$
\Delta \beta_{n} \equiv \beta_{0}-\beta_{n}
$$

so that $\Delta \omega$ and $\Delta n$ become the new free parameters. Typically, $\bar{\omega}$ is the center of the gain spectrum and $\bar{n}$ is the approximate threshold carrier density. To lowest order, we can ignore the appearance of $\Delta \omega$ and $\Delta n$ in the reflectivity matrices, but we must be more careful in the propagation matrix. We expand the diagonal elements of the matrix $\tilde{\tilde{B}} L$ in a Taylor series as

$$
\begin{aligned}
\beta_{n}(\omega, n) L= & \beta_{n}(\bar{\omega}, \bar{n}) L+\frac{\partial \beta_{0}}{\partial \omega} \Delta \omega L+\frac{\partial \beta_{0}}{\partial n} \Delta n L \\
& +\frac{\partial \Delta \beta_{n}}{\partial \omega} \Delta \omega L+\frac{\partial \Delta \beta_{n}}{\partial n} \Delta n L+\cdots .
\end{aligned}
$$

The last two terms of (29) are typically much less than 1 and can be dropped entirely. This assumption is tantamount to assuming that all of the longitudinal modes of the resonator are degenerate. The second and third terms are common to all elements of $\tilde{B} L$ and may be pulled out as a scalar times the identity $\tilde{\tilde{I}}$. The result is that the propagation matrix can be written as

$$
\begin{aligned}
\tilde{\tilde{P}}(\omega, n ; L) \approx & \exp \left(-j\left(\frac{\partial \beta_{0}}{\partial \omega} \Delta \omega+\frac{\partial \beta_{0}}{\partial n} \Delta n\right) L\right) \\
& \cdot \tilde{\tilde{P}}(\bar{\omega}, \bar{n} ; L) .
\end{aligned}
$$

With this substitution, (5) can be written as

$$
\left[\tilde{\tilde{R}}_{2} \tilde{\tilde{P}}(\bar{\omega}, \bar{n} ; L) \tilde{\tilde{R}} \tilde{P}^{\tilde{P}}(\bar{\omega}, \bar{n} ; L)-\gamma \tilde{\tilde{l}}\right] \tilde{A}=\tilde{0}
$$

where

$$
\gamma \equiv \exp \left(2 j\left(\frac{\partial \beta_{0}}{\partial \omega} \Delta \omega+\frac{\partial \beta_{0}}{\partial n} \Delta n\right) L\right) .
$$

Equation (31) is now a linear eigenvalue problem which can be solved for $\gamma$ by setting the determinant of the quantity in the square brackets equal to 0 .

This approximation is equivalent to that made by the Fox-Li iteration technique [20]; they both rely on the longitudinal cavity modes' degeneracy. From the form of (31), we see that $|\gamma|^{2}$ represents the fraction of energy left in the resonator after one roundtrip. In conventional unstable resonator theory, $|\gamma|^{-2}$ is the magnification $M$. For typical semiconductor lasers, the size of the matrix is around $100 \times 100$, which can be handled by most canned diagonalization routines. Often, only the few lowest loss modes are desired, and one can use an approximate technique (e.g., the Prony method [19]) to find them without actually diagonalizing the entire matrix. In many cases (as we will show in the next section), the lowest loss eigenvalues vary widely in magnitude, a property which increases the accuracy of such approximate techniques. The calculations presented in the remainder of this paper were

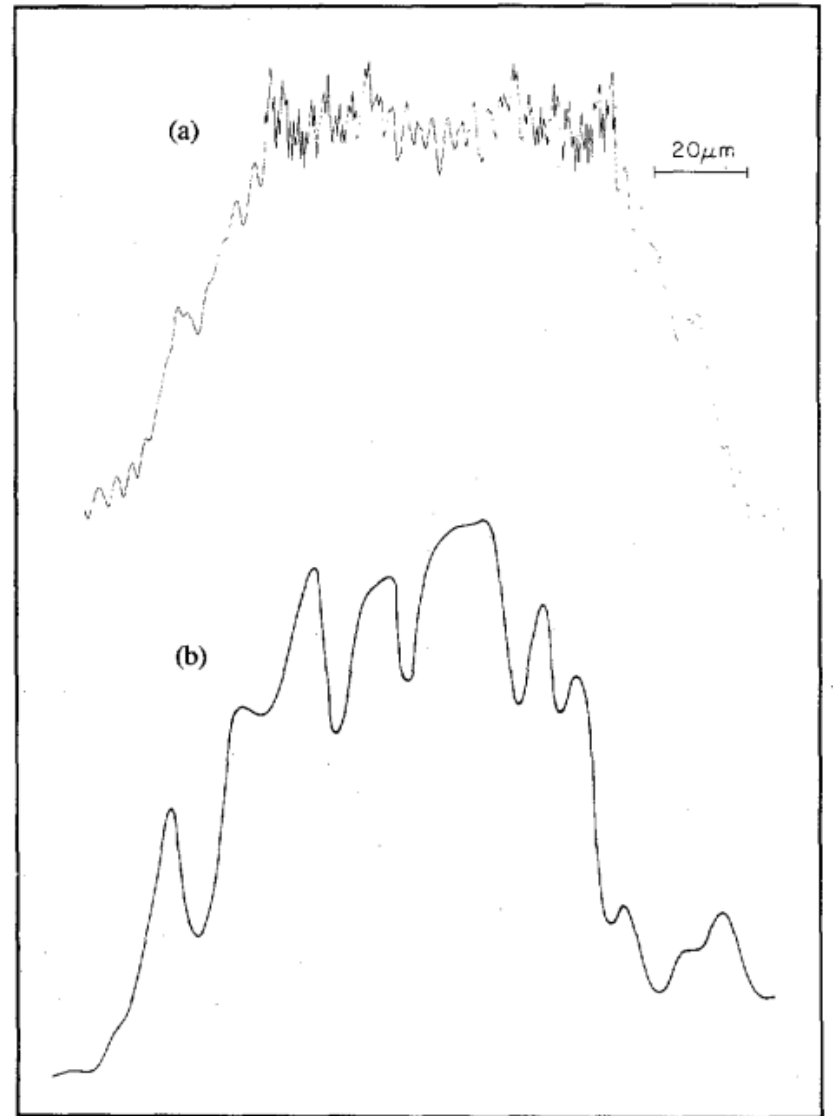

Fig. 3. Near field of a purely gain-guided URSL of width $80 \mu \mathrm{m}$, length $200 \mu \mathrm{m}$, and two symmetric mirrors of radius $250 \mu \mathrm{m}$. (a) Theoretical. (b) Experimental.

performed on a VAX 11/780 using root-finding and matrix routines from the IMSL subroutine library.

\section{EXAMPLES}

\section{A. Gain-Guided Unstable Resonator Semiconductor Laser}

Recently, we reported the operation of an unstable resonator semiconductor laser with pure gain guiding [8]. The near field as calculated using our model is shown in Fig. 3(a), while an experimental plot is shown in Fig. 3(b). The experimental and theoretical plots are both single valued (nodeless) over the same width (which is to be expected, the width being the gain stripe). The large modulation visible on the experimental plot we attribute to imperfections in the output mirror which scatters light and causes diffraction fringes. The roundtrip gain (normalized to that of plane wave propagation in the core material) was calculated to be $|\gamma|^{2}=0.286, M=3.5$. The geometric model of unstable resonators [9] for a symmetric, two-mirror resonator predicts

$$
M=1+\frac{L}{R}+\left[\frac{L^{2}}{R^{2}}+\frac{2 L}{R}\right]^{1 / 2} .
$$

For the laser of Fig. 3, $M=3.3$ according to the geometrical model (which, of course, neglects diffraction and loss in the cladding). One interesting feature of unstable 
resonators is the large difference in roundtrip losses between the lowest loss and next lossy resonator lateral mode. For the laser of Fig. 3, the next lossy mode has $|\gamma|^{2}=0.112$. In the absence of curved mirrors, the lateral waveguide modes are also the resonator modes, and an examination of the pole plot for the laser of Fig. 3 shows that there are well over 50 lateral waveguide modes with losses within a few percent of each other. The importance of large gain separation between the lowest loss resonator modes becomes clear when one takes into account small perturbations from the ideal waveguide (for example, resulting from defects or nonlinearities which appear at high pump currents). Consider a set of resonator modes $\left\{e_{i}(y\right.$, $z)\}$ with eigenvalues $\left\{\gamma_{i}\right\}$. Any imperfection in the waveguide will cause some small scattering between the modes; the scattering can be characterized by matrix elements $\left\{\kappa_{i j}\right\}$ (calculated in the same way as the set of $\left\{r_{i j}\right\}$ were). For small scattering, we can use first-order perturbation theory [22] to calculate the effects on the modes:

$$
e_{i}^{(1)}=e_{i}^{(0)}+\sum_{j \neq i} \frac{\kappa_{i j}}{\gamma_{i}-\gamma_{j}} e_{j}^{(0)} .
$$

As long as $\kappa_{i j}<<\gamma_{i}-\gamma_{k}$, the lasing mode in the presence of the perturbation will closely resemble the calculated mode in the absence of the perturbation. On the other hand, if the coupling approaches the order of magnitude of the separation between the gains of the $i$ th and $j$ th modes, the modes will become mixed by the perturbation (also, the perturbation theory breaks down). Consequently, there is poor mode selection in flat-mirror broad lasers, and small perturbations in the optical cavity due to nonlinearities which cause the laser to jump among mixtures of the nearly degenerate lateral modes. The strong coupling imposed by the curved mirrors of a URSL, however, breaks this degeneracy; the lowest order mode is strongly preferred and is relatively insensitive to small perturbations to the cavity. This explains the stability of the single lateral resonator mode that was observed up to four times threshold.

Another feature of interest is the variation of mirror loss per bounce with numerical aperture. Fig. 4 shows the loss per bounce as a function of waveguide width with all other parameters held fixed. As we vary the width, we are increasing the equivalent Fresnel number. The periodic mode crossings observed by Fox and $\mathrm{Li}[20]$ in openwalled unstable resonator structures are apparent.

\section{B. Unstable Resonator with Lateral Waveguiding}

One of the drawbacks to URSL's is that the lossy material in the cladding absorbs the diffraction losses of the resonator (which, in many other systems, are useful output) and degrades the external quantum efficiency. In fact, by extension of existing formulas, one can show that the external quantum efficiency is given by [8], [23]

$$
\frac{1}{\eta_{d}}=\frac{1}{\eta_{i}}\left(1+\frac{\alpha L}{\ln \frac{1}{R}+\ln \frac{1}{|\gamma|}}\right) \frac{1-|\gamma| R}{1-R}
$$

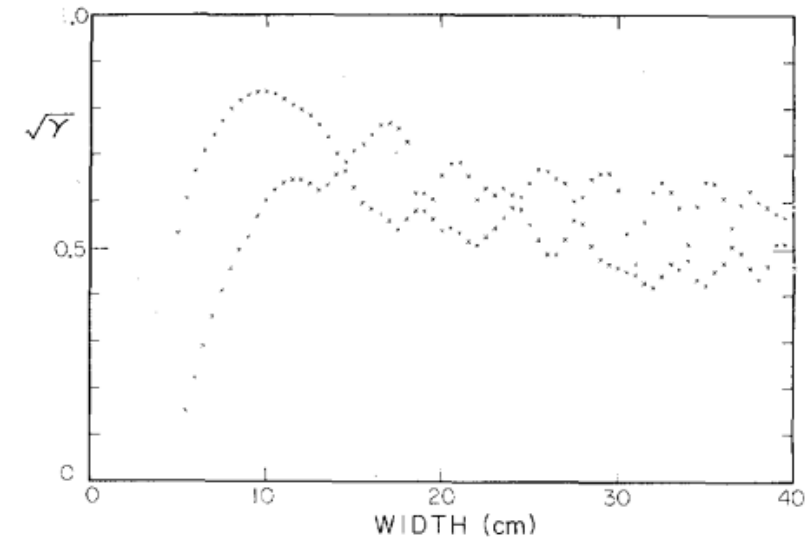

Fig. 4. Amplitude loss per bounce $(\sqrt{\gamma})$ versus width for two lowest loss modes of a symmetric gain-guided URSL of length $200 \mu \mathrm{m}$, mirror radius $-250 \mu \mathrm{m}$. $\gamma$ gives the roundtrip amplitude loss, $\gamma^{2}$ gives the roundtrip energy loss. Calculations performed at $0.5 \mu \mathrm{m}$ intervals.

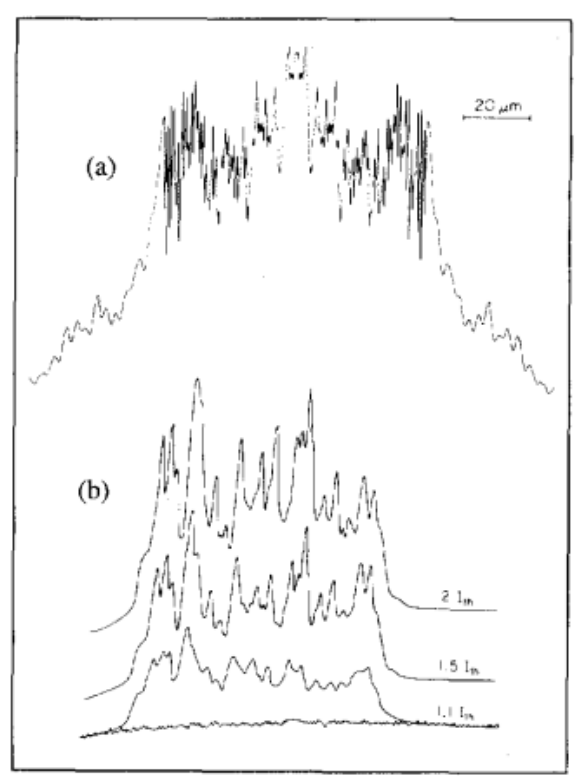

Fig. 5. Near field of an index-guided URSL (same dimensions as Fig. 4) with a real index step of 0.03 . (a) Theoretical. (b) Experimental.

where $\alpha$ is the loss in the unpumped medium, $R$ is the mirror reflectivity, and $\eta_{i}$ is the internal quantum efficiency. (In [8], a factor $M$ is missing from the numerator of the equivalent expression.) One suggested method for increasing the quantum efficiency of a URSL is to add a real index step in the lateral direction to provide optical confinement [12]. Fig. 5(a) and (b) shows the calculated and measured near fields, respectively, of such a structure. As in the simple URSL, one gets a near field with an approximately constant average power density across the gain stripe; only in this case, there is high-frequency, high-modulation-depth spatial modulation on the near field due to the interference of the reflections off the confining walls. The spatial frequencies evident in the theoretical plot are greater due to the limited resolution of the experimental viewing system. This structure combines the advantages of a Fabry-Perot broad area laser and a purely gain-guided URSL, namely, the high quantum efficiency 


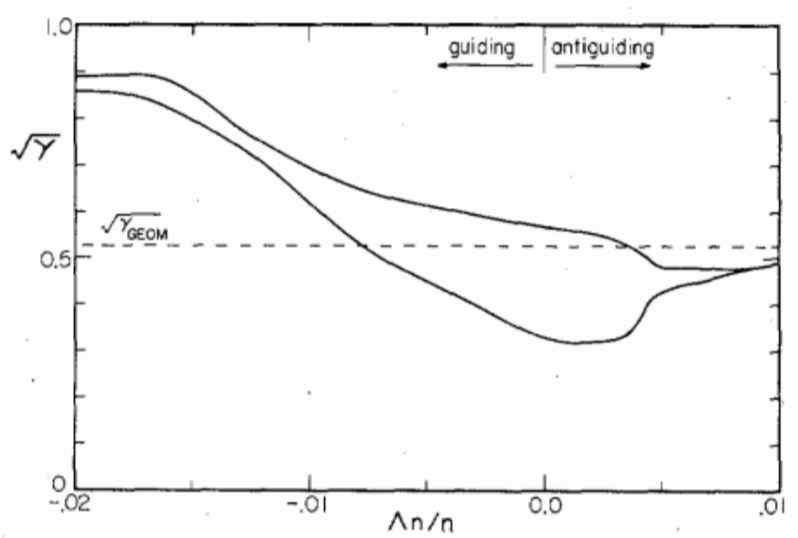

Fig. 6. Roundtrip losses for the two lowest order modes as a function of real index step. $\Delta n$ is the difference in index between the cladding and core so that the left portion of the graph corresponds to a "guiding" waveguide and the right portion corresponds to an "antiguiding"' waveguide. The dotted line indicates the value of $\sqrt{\gamma}$ one would calculate using the geometric theory of unstable resonators.

of the former and the stable near field of the latter. The results suggest that a larger index step is more desirable from the point of view of lowering losses, and in Fig. 6 we have plotted the losses of the two lowest loss modes as a function of index step. Although the losses continue to improve with larger index step, the gain separation between the two lowest loss modes decreases at the same time (also, the high spatial frequency ripples evident in Fig. 5 become larger and larger, which increases the sidelobes in the far field). Thus, there is an optimum index step which brings about low losses while maintaining high gain separation between modes.

\section{Tilted-Mirror Resonators}

Not all high-loss resonators possess circular mirrors. For example, two misaligned planar mirrors make up a tilted-mirror resonator. This geometry is interesting from the geometrical optics picture because, unlike classical unstable and stable resonators, there is no ray within the resonator which reproduces itself. This property suggests that incipient filaments within the resonator will also be unable to reproduce themselves. (It also suggests that the losses will be rather high.) Recently, we demonstrated operation of a tilted-mirror semiconductor laser [13]; the lowest loss mode calculated using the foregoing analysis (with $\Delta l(y) \equiv y \tan \theta$, with $\theta$ the tilt angle at one mirror) and an experimental near field measurement of the device are reproduced in Fig. 7(a) and (b), respectively. In performing the calculation, using a uniform gain distribution under the stripe contact yields two nearly degenerate resonator modes. However, we observed in the subthreshold near field of a $0^{\circ}$ laser that there was slightly higher gain at the edges of the stripe which favored the theoretical mode shown in Fig. 7. One of the disadvantages of the tilted-mirror design is that the losses are quite high (since the only part of the resonator mode that reproduces itself comes from diffraction). Loss as a function of tilt angle is given in Fig. 8. The decrease in loss at large angle is interesting since it suggests a region of low loss around

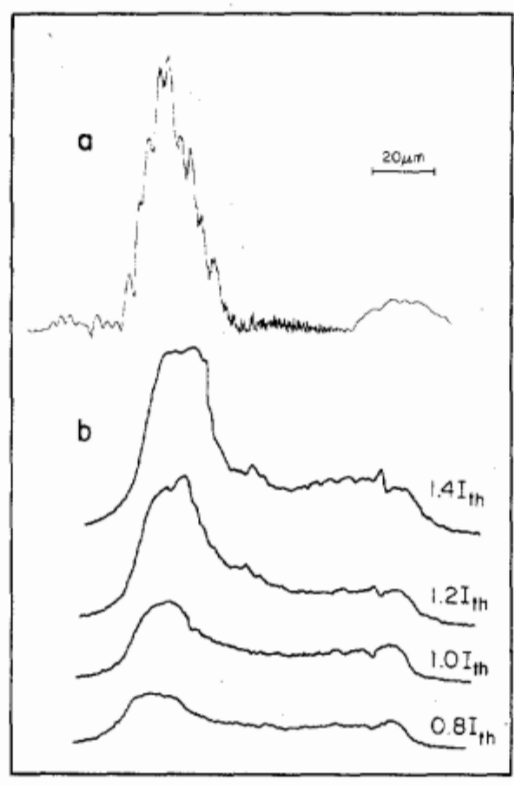

Fig. 7. Near field of a tilted-mirror laser of width $100 \mu \mathrm{m}$, length $300 \mu \mathrm{m}$, tilt angle $10^{\circ}$. (a) Theoretical. (b) Experimental.

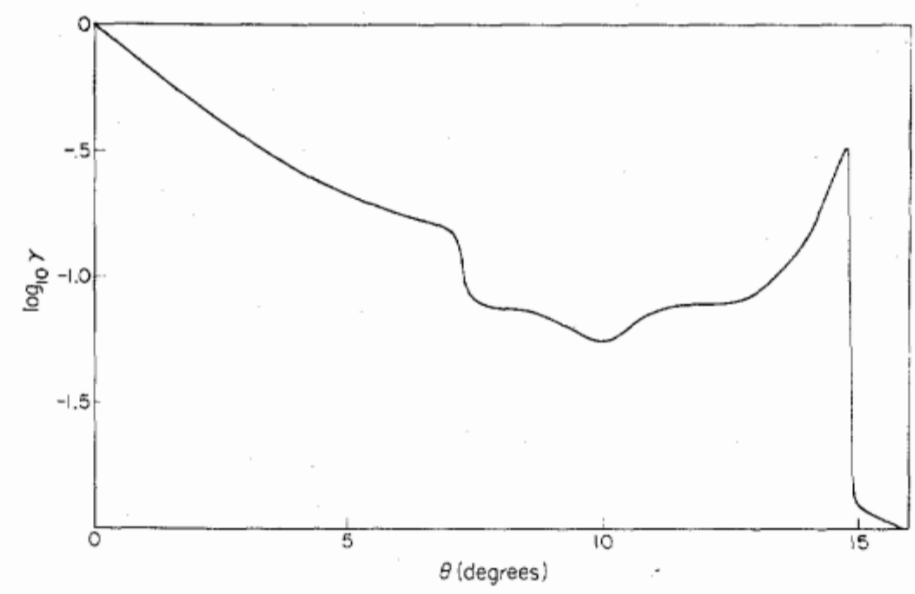

Fig. 8. Roundtrip energy loss as a function of tilt angle for a tilted-mirror resonator of same dimensions as Fig. 7.

$13-14^{\circ}$ (unfortunately, the devices fabricated in [13] neatly bracketed this region-in fact, the losses at $0^{\circ}, 5^{\circ}$, $10^{\circ}$, and $15^{\circ}$ all lie neatly on a straight line). Such a "loss window" was recently observed in calculations of tilt effects in $\mathrm{CO}_{2}$ waveguide lasers [24].

\section{Conclusions}

We have performed an analysis of semiconductor lasers with nonplanar mirrors that includes the effects of lateral gain/index guiding. We developed a criterion for truncation of the infinite set of waveguide modes based on the analytic structure of the Green's function for the waveguide problem. Then, using a thin-lens approximation, the mirrors were shown to couple the waveguide modes. We then reduced the nonlinear equation for frequency and gain to a linear eigenvalue problem where the magnitude of the eigenvalues gave the cold-cavity losses. We applied the analysis to three cases of interest-a gain-guided URSL, an index-guided URSL, and a gain-guided tilted-mirror resonator. The calculated near fields agreed with those of 
measured devices. We also showed that URSL's .possess high gain separation between the lowest loss modes. The addition of a lateral index waveguide can reduce the losses while maintaining gain separation and resistance to filamentation at the expense of degrading the smoothness of the near field. Tilted-mirror resonators were shown to possess well-defined modes, and the variation of loss with tilt angle was presented.

\section{REFERENCES}

[1] R. D. Burnham, D. R. Scifres, W. Streifer, and S. Peled, "Nonplanar large optical cavity GaAs/GaAlAs semiconductor laser," Appl. Phys. Lett., vol. 35, pp. 734-736, 1979.

[2] D. Scifres, R. D. Burnham, and W. Streifer, "Phase-locked semiconductor laser array," Appl. Phys. Lett., vol. 33, pp. 1015-1017, 1978.

[3] D. Scifres, C. Lindstrom, R. D. Burnham, W. Streifer, and T. L. Paoli, "Phase-locked (GaAl)As laser diode emitting $2.6 \mathrm{~W} \mathrm{CW}$ from a single mirror," Electron. Lett., vol. 19, pp. 169-171, 1983.

[4] H. Temkin, R. A. Logan, J. P. van der Ziel, C. L. Reynolds, and S. M. Tharaldsen, "Index-guided arrays of Schottky barrier confined lasers," Appl. Phys. Lett., vol. 46, pp. 465-467, 1985.

[5] D. Scifres, W. Streifer, R. D. Burnham, and T. L. Paoli, "Near field and far-field patterns of phase-locked semiconductor laser arrays," Appl. Phys. Lett., vol. 42, pp. 495-497, 1983.

[6] A. P. Bogatov, P. G. Eliseev, M. A. Man'ko, G. T. Mikaelyan, and Yu. M. Popov, "Injection laser with an unstable resonator," Sov. J. Quantum Electron., vol. 10, pp. 620-622, 1980

[7] R. R. Craig, L. W. Casperson, O. M. Stafssudd, J. J. J. Yang, G. A. Evans, and R. A. Davidheiser, "High loss resonators for semiconductor diode lasers," Electron. Lett., vol. 21, pp. 62-63, 1985.

[8] J. Salzman, T. Venkatesan, R. Lang, M. Mittlestein, and A. Yariv, "Unstable resonator cavity semiconductor lasers," Appl. Phys. Lett., vol. 46, pp. 218-220, 1985 .

[9] A. E. Siegman, "Unstable optical resonators for laser applications," Proc. IEEE, vol. 53, pp. 277-287, 1965.

[10] P. Horwitz, "Asymptotic theory of unstable resonator modes," $J$. Opt. Soc. Amer., vol. 63, pp. 1528-1543, 1983.

[11] L.-W. Chen and L. B. Felsen, "Coupled-mode theory of unstable resonators," IEEE J. Quantum Electron., vol. QE-9, pp. 1102-1113, 1973.

[12] J. Salzman, R. Lang, M. Mittlestein, and A. Yariv, "Modal prop- erties of unstable resonators with a lateral waveguide," Appl. Phys Lett., vol. 47, pp. 445-447, 1985.

[13] J. Salzman, R. Lang, S. Margalit, and A. Yariv, "Tilted mirror semiconductor lasers," Appl. Phys. Lett., vol. 47, pp. 9-11, 1985.

[14] W. Streifer and E. Kapon, "Application of the equivalent-index method to DH diode lasers," Appl. Opt., vol. 18, pp. 3724-3725, 1979.

[15] D. Marcuse, Theory of Dielectric Optical Waveguides. New York: Academic, 1974 , p. 1 .

[16] B. Freidman, Principles and Techniques of Applied Mathematics. New York: Wiley, 1956, p. 214.

[17] J. W. Goodman, Introduction to Fourier Optics. New York: McGraw-Hill, 1968, p. 77.

[18] M. Abramowitz and I. A. Stegun, Handbook of Mathematical Functions. Washington, DC: Nat. Bur. Standards, 1964, p. 295

[19] A. E. Siegman and H. Y. Miller, "Unstable optical resonator loss calculations using the Prony method," Appl. Opt., vol. 9, pp. 2729$2736,1970$.

[20] A. G. Fox and T. Li, "Resonant modes in a master interferometer," Bell Syst. Tech. J., vol. 40, pp. 453-488, 1961.

[21] L. Bergstein, "Modes of stable and unstable optical resonators," Appl. Opt., vol. 7, pp. 495-504, 1968.

[22] C. Cohen-Tannoudji, B. Diu, and F. Laloe, Quantum Mechanics, Vol. II. New York: Wiley, 1977, p. 1108.

[23] J. Salzman, R. Lang, and A. Yariv, "Efficiency of unstable resonator semiconductor lasers,"' Electron. Lett., vol. 21, pp. 821-823, 1985.

[24] C. A. Hill and D. R. Hall, "Mirror tilt effects in waveguide laser resonators," presented at Conf. Lasers and Electro-Opt., Baltimore, MD, May 1985, paper FN6.

Robert J. Lang ( $\mathrm{S}^{\prime} 83$ ) for a photograph and biography, see this issue, $\mathrm{p}$. 448.

Joseph Salzman, photograph and biography not available at the time of publication.

Amnon Yariv (S'56-M'59-F'70) for a photograph and biography, see this issue, p. 448 . 